\title{
Immediate Partial Breast Reconstruction with Endoscopic Latissimus Dorsi Muscle Flap Harvest
}

\author{
Chae Eun Yang ${ }^{1,2}$, Tai Suk Roh ${ }^{1,2}$, In Sik Yun ${ }^{1,2}$, Young Seok Kim ${ }^{1,2}$, Dae Hyun Lew ${ }^{1,2}$ \\ ${ }^{1}$ Department of Plastic and Reconstructive Surgery, Gangnam Severance Hospital, Yonsei University College of Medicine, Seoul; ${ }^{2}$ Institute for \\ Human Tissue Restoration, Yonsei University College of Medicine, Seoul, Korea
}

Background Currently, breast conservation therapy is commonly performed for the treatment of early breast cancer. Depending on the volume excised, patients may require volume replacement, even in cases of partial mastectomy. The use of the latissimus dorsi muscle is the standard method, but this procedure leaves an unfavorable scar on the donor site. We used an endoscope for latissimus dorsi harvesting to minimize the incision, thus reducing postoperative scars.

Methods Ten patients who underwent partial mastectomy and immediate partial breast reconstruction with endoscopic latissimus dorsi muscle flap harvest were reviewed retrospectively. The total operation time, hospital stay, and complications were reviewed. Postoperative scarring, overall shape of the reconstructed breast, and donor site deformity were assessed using a 10-point scale.

Results In the mean follow-up of 11 weeks, no tumor recurrence was reported. The mean operation time was $294.5( \pm 38.2)$ minutes. The postoperative hospital stay was 11.4 days. Donor site seroma was reported in four cases and managed by office aspiration and compressive dressing. Postoperative scarring, donor site deformity, and the overall shape of the neobreast were acceptable, scoring above 7.

Conclusions Replacement of 20\% to $40 \%$ of breast volume in the upper and the lower outer quadrants with a latissimus dorsi muscle flap by using endoscopic harvesting is a good alternative reconstruction technique after partial mastectomy. Short incision benefits from a very acceptable postoperative scar, less pain, and early upper extremity movement.

Keywords Mammaplasty / Endoscopes / Surgical flaps
Correspondence: Young Seok Kim

Department of Plastic and

Reconstructive Surgery, Yonsei

University College of Medicine,

211 Eonju-ro, Gangnam-gu,

Seoul 135-720, Korea

Tel: +82-2-2019-3600

Fax: +82-2-2019-4833

E-mail: psyskim@yuhs.ac

This article was presented at the the $3 \mathrm{rd}$ Research and Reconstructive Forum on May 10, 2013, in Dagu, Korea.

No potential conflict of interest relevant to this article was reported.

Received: 2 Apr 2014 • Revised: 11 May 2014 • Accepted: 31 May 2014

pISSN: 2234-6163 • elSSN: 2234-6171 • http://dx.doi.org/10.5999/aps.2014.41.5.513 • Arch Plast Surg 2014;41:513-519

\section{INTRODUCTION}

Improvement of diagnostic techniques and widespread breast cancer screening campaigns have made early detection of breast cancer possible [1]. Partial mastectomy followed by radiation therapy is usually recommended for patients with early-stage breast cancer with an operable locoregional tumor. This is re- ferred to as breast conservation therapy. Depending on the volume excised, significant contour deformities could occur. To compensate for the deformity, volume displacement or volume replacement could be considered for optimal cosmetic results $[2,3]$. For Koreans, whose breast volume is usually small to moderate [4], which implies a high tumor to breast ratio, the volume replacement procedure is more commonly performed 
than volume displacement in order to match the opposite breast [2]. The use of the latissimus dorsi muscle flap is the standard method for replacement $[3,5]$. Although this flap is reliable and its elevation is technically straightforward, it has one drawbackthis surgery leaves a long horizontal scar on the donor site. Adams et al. [6] reported that $22 \%$ of the patients who underwent a transfer of the latissimus dorsi muscle for unilateral breast reconstruction rated their scar as unacceptable. To maximize the cosmetic satisfaction, a minimally invasive harvest technique has been devised. In Japan in 1995, video-assisted skin-sparing partial mastectomy and immediate reconstruction with a latissimus dorsi pure muscle flap was introduced. In 1997, Cho et al. [7] reported a free latissimus dorsi muscle transfer with an endoscope. In 2007, Missana and Pomel [5] reported endoscopic harvesting of the latissimus dorsi muscle for immediate breast reconstruction after a skin-sparing mastectomy. Some surgeons have introduced flap harvesting through a short incision without an endoscope [8]. Further, in 2012, a robotic latissimus dorsi muscle harvest technique was reported [9]. All of them appear to offer an oncologically safe method with high patient satisfaction. However, despite the increasing popularity of breast conservation therapy, there are only few reports on immediate partial breast reconstruction with a partial latissimus dorsi muscle flap harvested using an endoscope. In this study, we present our experience of ten patients who underwent immediate partial breast reconstruction with an endoscope-assisted latissimus dorsi muscle flap after partial mastectomy. We reviewed them and noted the surgical complications and oncological safety.

\section{METHODS}

Ten patients were reviewed in this study in the time period of June 2011 to March 2013. All the tumors were located in the upper-outer or the lower-outer quadrant, and all of the patients underwent partial mastectomy by a general surgeon (Fig. 1A). Intraoperative frozen section analysis for margin assessment and sentinel lymph nodes was performed in all cases. After partial mastectomy, while patients remained in a supine position, the thoracodorsal pedicle was identified and the thoracodorsal nerve was ligated. The origin and some of the lateral portion of the latissimus dorsi muscle was dissected via mastectomy and sentinel lymph node biopsy incisions. Then, in order to determine the volume needed, laparotomy sponges were packed into the defect and trimmed off to match the symmetry with the opposite breast. Next, the patient's position was changed to lateral decubitus with the upper arm on an armrest in a slightly abducted position. Pediatric Omni-tract retractors (Integra, Plainsboro, NJ, USA) with a customized curvilinear retractor were placed

Fig. 1. Partial breast reconstruction with endoscopic latissimus dorsi muscle flap harvest

A 41-year-old female with a left lower quadrant tumor on the left breast. (A) After partial mastectomy and sentinel lymph node biopsy. (B) Placement of Endoscopic instruments. (C) Harvested latissimus dorsi muscle at a lateral decubitus position and (D) at a sitting position. (E) Immediately after partial breast reconstruction at a sittign position.
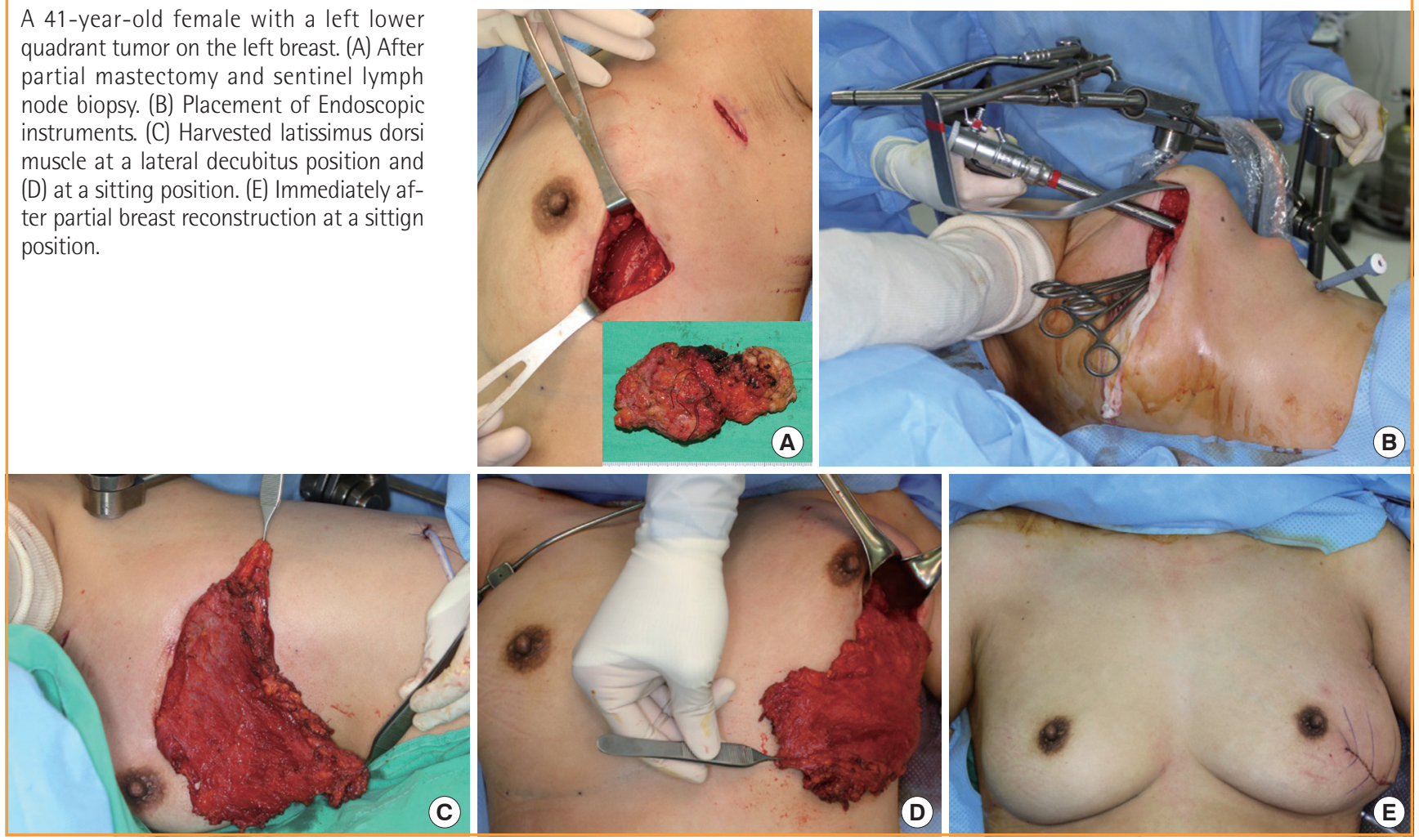
through the mastectomy incision. Only a small incision was added on the flan for the trocar insertion (Fig. 1B). An endoscope (Richard Woolf, Knittlingen, Germany) was introduced through the main incision site, and further dissection was carried out. First, with the gentle traction of the latissimus dorsi muscle using Allis clamps, suprafascial dissection was performed between the skin and the latissimus dorsi muscle, followed by submuscular dissection using an endoscopic monopolar dissector. After complete dissection above and below the latissimus dorsi muscle, an appropriately sized muscle flap was designed. Mobilization of the muscular origin and insertion was done using a harmonic endoscopic scalpel (Harmonic Ace, Ethicon, Blue Ash, OH, USA). Negative suction drains were inserted via the trocar insertion site. A flap was inset into the defect area while the patients were in a sitting position, and closure was done layer by layer (Fig. 1C-E).

The patients' body mass index (weight $[\mathrm{kg}] /$ height $\left[\mathrm{m}^{2}\right]$ ) was calculated, and the total operation time was recorded. The postoperative hospital stay was also recorded, and the postoperative complications were reviewed with the patients' medical records. The postoperative scar was assessed by using the Patient and Observer Scar Assessment Scale. The patients were asked to record their score on the patient scale questionnaire of the Patient and Observer Scar Assessment Scale 3 months after the operation. The observer scale was scored using photographs. Each item of both scales had a 10-point score, with 10 indicating the worst imaginable scar or sensation and 1 corresponding to the situation of normal skin. The overall shape of the reconstructed breast and the donor site deformity were also scored using the 10-point scale, from very dissatisfied to very satisfied in an ascending manner.

\section{RESULTS}

The mean follow-up period was 11 weeks ( 3 weeks to 6 months). The patients were in the age group of $41.2 \pm 7.1$ years, and their body mass index was $21.9 \pm 2.5 \mathrm{~kg} / \mathrm{m}^{2}$. All the tumors were located in the outer quadrant of the breast (Table 1). The nippleareolar complex and the overlying skin were preserved during mastectomy. The intraoperative frozen section analyses of the tumor margin and the sentinel lymph nodes were all negative. The mean operation time was $294.5 \pm 38.2$ minutes; it became shorter as the surgeon became more experienced with the procedure. The mean postoperative hospital stay was 11.4 days (range, 7-16 days). There were no donor site complications such as intraoperative bleeding, postoperative hematoma, or infection, except for

\section{Table 1. Patient demographics}

\begin{tabular}{cccccl}
\hline No. & Age $(\mathbf{y r})$ & BMI $\left(\mathbf{k g} / \mathbf{m}^{2}\right)$ & Tumor location & Excised tumor $(\mathbf{g})$ & \multicolumn{1}{c}{ Tumor histology } \\
\hline 1 & 42 & 22.9 & LLQ, Lt. & 48 & Invasive ductal carcinoma \\
2 & 50 & 22.4 & LUQ, Lt. & 50 & Ductal carcinoma in situ \\
3 & 52 & 26.2 & RLQ, Rt. & 75 & Invasive ductal carcinoma with ductal carcinoma in situ \\
4 & 41 & 21.9 & LLQ, Lt. & 46 & Ductal carcinoma in situ \\
5 & 26 & 17.5 & RUQ, Rt. & 50 & Invasive ductal carcinoma \\
6 & 42 & 24.6 & LUQ, Lt. & 82 & Invasive lobular carcinoma \\
7 & 43 & 20.0 & LUQ, Lt. & 100 & Invasive ductal carcinoma \\
8 & 38 & 20.5 & RUQ, Rt. & 100 & Invasive ductal carcinoma with ductal carcinoma in situ \\
9 & 37 & 20.4 & LUQ, Lt. & 53 & Invasive ductal carcinoma with ductal carcinoma in situ \\
10 & 41 & 22.4 & RUQ, Rt & 65 & Ductal carcinoma in situ \\
\hline
\end{tabular}

BMI, body mass index; LLQ, left lower quadrant; Lt., left side; RLQ, right lower quadrant; Rt., right side; LUQ, left upper quadrant; RUQ, right upper quadrant.

\section{Table 2. Surgical outcomes}

\begin{tabular}{rcclc}
\hline No. & $\begin{array}{c}\text { Operation time for } \\
\text { reconstruction ( } \mathbf{m i n})\end{array}$ & $\begin{array}{c}\text { Hospital } \\
\text { stay (day) }\end{array}$ & Complications & $\begin{array}{c}\text { Postoperative } \\
\text { radiotherapy (Gy) }\end{array}$ \\
\hline 1 & 280 & 15 & Donor site seroma & 50.4 \\
2 & 325 & 9 & None & 50.4 \\
3 & 300 & 10 & None & 50.4 \\
4 & 16 & Donor site seroma & 50.4 \\
5 & 350 & 13 & Donor site seroma & 59.4 \\
6 & 250 & 11 & Donor site seroma & 113.4 \\
7 & 340 & 12 & None & 50.4 \\
8 & 240 & 7 & None & 50.4 \\
10 & 270 & 14 & None & 59.4 \\
\hline
\end{tabular}




\section{Fig. 2. Six months after radiation on reconstructed breast}

A 37-year-old female 6 months after immediate partial breast reconstruction with a latissimus dorsi muscle flap harvested using an endoscope after partial mastectomy $(53 \mathrm{~g})$ due to left side left upper quadrant breast cancer. 59.4-Gy radiation therapy followed on the left breast. The volume was acceptably replaced with only a curvilinear scar on the left breast. (A) Anterior view. (B) Posterior view.

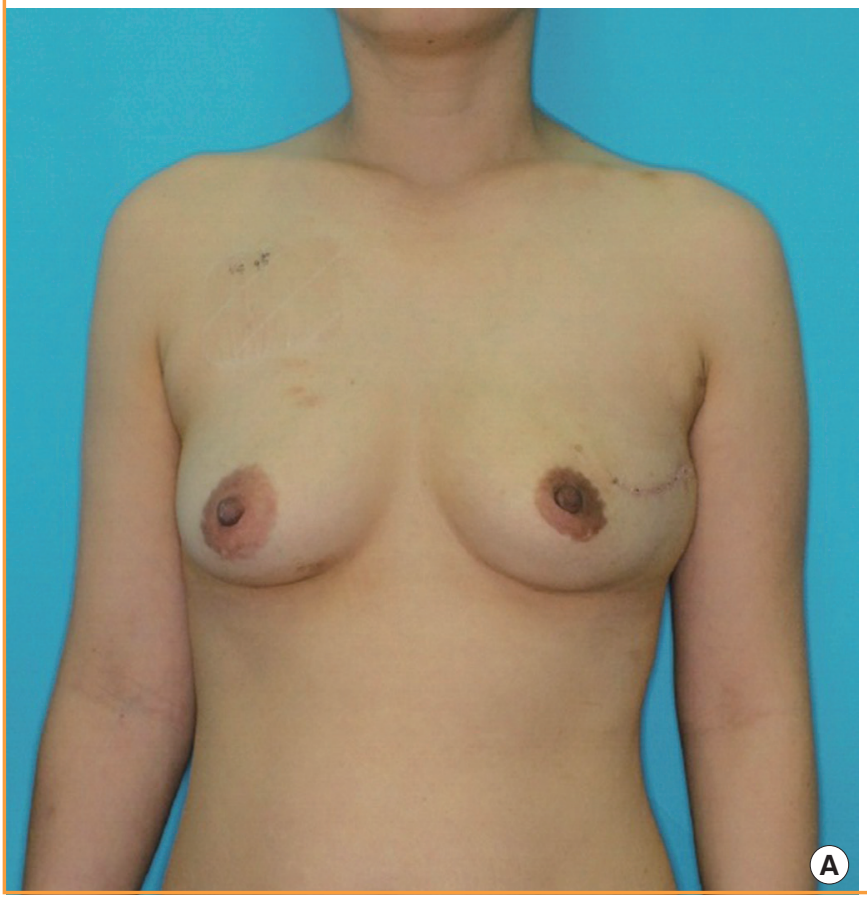

Table 3. The patient and observer scar assessment scale

\begin{tabular}{|lc|}
\hline Items & Average score ( \pm standard deviation) \\
\hline Patient scale & \\
Pain & $1.1 \pm 0.18$ \\
Itching & $1.3 \pm 0.48$ \\
Color & $3.9 \pm 0.94$ \\
Stiffness & $2.1 \pm 0.54$ \\
Thickness & $2.1 \pm 0.54$ \\
Irregularity & $2.2 \pm 0.68$ \\
Overall & $3.2 \pm 0.92$ \\
Observer scale & \\
Vascularity & $2.8 \pm 0.64$ \\
Pigmentation & $4.5 \pm 1.2$ \\
Thickness & $2.8 \pm 1.0$ \\
Relief & $2.4 \pm 0.68$ \\
Pliability & $2.5 \pm 0.7$ \\
Surface area & $2.7 \pm 0.7$ \\
Overall & $3.5 \pm 1.1$ \\
\hline
\end{tabular}

seroma formation, which developed in four cases (Table 2). This was managed by office aspiration and compressive dressings at 5 weeks.

Among the postoperative scars, only the one on the mastectomy site was easily detectable (Fig. 2). The axillary scar was hidden, and the trocar insertion site scar was barely noticeable. The overall Patient and Observer Scar Assessment Scale score was $3.2 \pm 0.92$, as recorded by the patients (Table 3 ), and $3.5 \pm 0.7$,

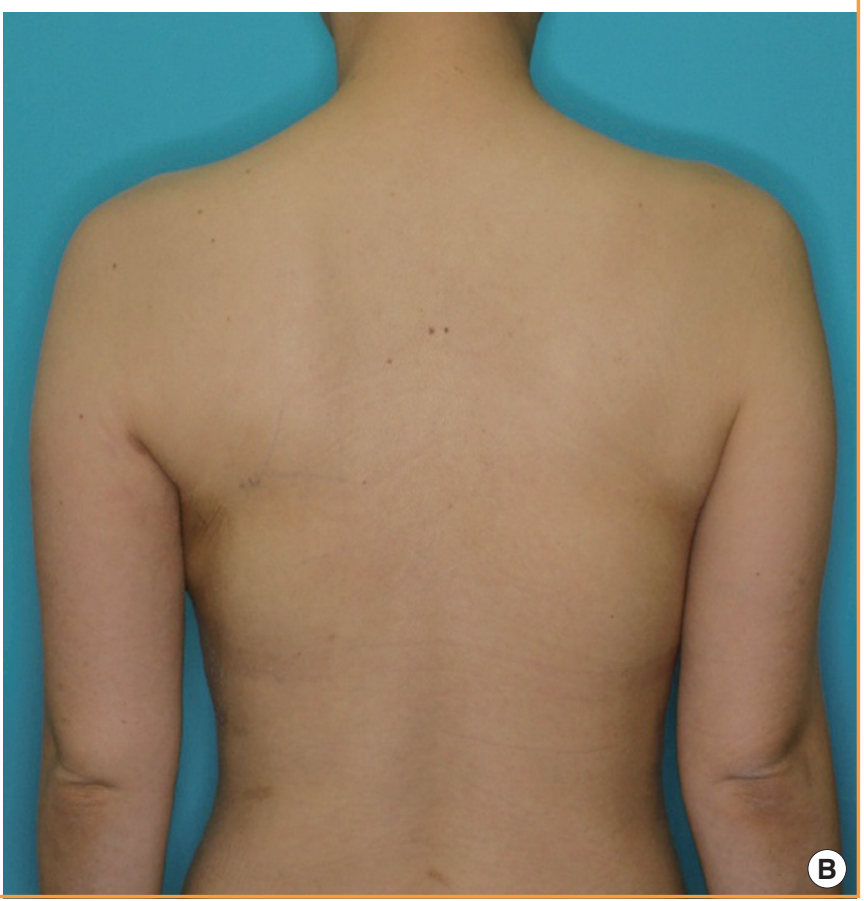

as recorded by the observer (Table 3 ).

Postoperative radiation of 50.4-113.4 Gy was administered in all cases (Table 2). After radiation therapy, the reconstructed breast shrunk slightly, but the final shape was acceptably maintained (Fig. 2). The patients scored the overall shape as $8.1 \pm$ 0.72 , and the observer scored it as $7.8 \pm 0.67$, on average. Donor site deformity was also acceptable, with a score of $7.8 \pm 0.68$ by the patients and $7.4 \pm 0.60$ by the observer (Table 3 ). During the follow-up period, no evidence of tumor recurrence was reported.

\section{DISCUSSION}

According to the database of the Centers for Disease Control and Prevention, breast cancer is the most common cancer and the second most common cause of cancer death among women of all races. In 2010, nearly 1.5 million people were diagnosed with breast cancer. The development of diagnostic techniques and an increased interest in breast cancer have made the early detection of breast cancer easier. Further, the use of breast conservation therapy (partial or segmental mastectomy/lumpectomy following adjuvant radiation) for the treatment of early-stage breast cancer is becoming increasingly popular. In the 2007 report of the National Cancer Institute breast cancer database, the proportion of breast conservation therapy in breast cancer sur- 
gery has increased from $40 \%$ in 1991 to $60 \%$ in 2002 . Oncoplastic surgery aims to achieve the best cosmetic outcome without any oncological compromise. In terms of oncological safety, a randomized study comparing breast conservation surgery with radical mastectomy for breast cancer shows a similar survival rate [10-12]. However, tumor removal can produce disfigurement of the breast, commonly leading to the patient's psychological vulnerability. The general trend is to reconstruct the defects immediately after mastectomy and before irradiation so as to avoid surgery on a scarred and contracted breast. As margins are the main concern for determining the reconstruction timing (whether immediate or delayed), confirmation of intraoperative frozen sections is an essential procedure. Preoperative magnetic resonance imaging would be helpful, as it shows no difference when compared with the pathological specimen [13].

Unfavorable cosmetic results following breast conservation surgery are related to breast shape, tumor size, tumor location, and postoperative radiation [12]. Central or lower quadrant tumors have worse cosmetic results than lumpectomies in other quadrants. The tumor to breast ratio is one of the most important factors when predicting the potential for a poor cosmetic result.

Studies have shown a decline in cosmetic satisfaction for patients with parenchymal resection [2] greater than $70-100 \mathrm{~cm}^{3}$ or for patients from whom more than $20 \%$ of the breast is excised [12]. Further, traditionally, women with large breasts have been thought to have increased complications, greater radiationinduced fibrosis and worse cosmetic outcomes after breast conservation therapy $[14,15]$.

To match the opposite breast, volume displacement or replacement is considered. The size of the tumor with respect to the volume of the breast is the deciding factor. For a small to moderately sized breast, as in most East Asian women [4], the volume replacement procedure is generally recommended for reconstruction. This can be performed by local tissue rearrangement or flap surgery depending on the tumor size and location. Less than $10 \%$ of a breast defect on the lateral side can be closed with local fasciocutaneous flaps. However, patients with large breast-to-tumor ratios have insufficient residual breast tissue after partial mastectomy, thus needing non-breast tissue to fill the defect. The latissimus dorsi muscle flap is now a standard reconstructive method for those patients $[3,5]$. The latissimus dorsi muscle has an excellent blood supply, wide dimensions, and long pedicle, and muscle harvesting is relatively easy. However, classic latissimus dorsi muscle harvesting leaves a long horizontal scar on the donor site. An unacceptable scar can detract from otherwise excellent reconstructive results.

As a response to concerns about the reduction of scarring, procedures that minimize postoperative scars have gradually be- come more popular among reconstructive surgeons. Endoscopic latissimus dorsi harvesting was first reported by Friedlander and Sundin [16] in 1994 in studies of cadavers. Three years later, Fine et al. [17] and Cho et al. [7] reported endoscopic harvesting of the latissimus dorsi muscle for covering lower limb defects. The open technique was used for the dissection of the muscle in the proximal part, and the distal part of the muscle was dissected with the closed technique by means of endoscopy. Cho et al. [7] reported that endoscopic harvesting was reproducible and feasible. Missana and Pomel [5] then reported endoscopic muscular latissimus dorsi flap harvesting after skin sparing mastectomy. This method uses a mastectomy incision and involves making an additional $3-\mathrm{cm}$ vertical incision on the flank. Following the Japanese development of video-assisted total mastectomy in 1995, video-assisted breast-conserving surgery is now commonly performed in Japan. It is indicated for the patients with no skin infiltration. Depending on the tumor location, a midaxillary or periareolar incision is made. Then skin-sparing partial mastectomy and simultaneous breast reconstruction are performed. Some surgeons have introduced flap harvesting through a short incision without an endoscope. According to their report [8], the lengths of the skin incisions made in endoscopic techniques are not significantly shorter than those of the short incision method. In 2012, Selber et al. [9] reported a robotic latissimus dorsi muscle harvest for three immediate and two delayed reconstructions after nipple-areola complex-sparing mastectomies with the advantage of high-resolution and more precise instrumentation.

In this study, we introduced an endoscope-assisted muscle harvesting method after partial mastectomy preserving the overlying skin envelope. Unlike the early methods that Fine et al. [17] and Cho et al. [7] have addressed, the defect site is near the donor site so that the mastectomy incision could be used as the main incision. A customized retractor is placed via the main incision and provides a more optimal visual field of the curvilinear pocket from the anterior chest wall to the midline of the back. A sentinel lymph node biopsy incision is also used for dissection of the muscle origin and thoracodorsal pedicle. We dissected the subcutaneous tissue first for easier submuscular dissection. Only one incision was added on the flank for trocar insertion. Dissection was performed with a mixed open and closed technique, and the muscle attachment was separated with a harmonic endoscalpel. Using the harmonic scalpel also helps simplify submuscular dissection. Among the postoperative scars, only the one on the mastectomy site is easily detectable. The axillary and trocar insertion site scars are barely noticeable.

To prevent unwanted postoperative animation, we ligated the thoracodorsal nerve. After radiation therapy, because the latissi- 
mus dorsi muscle is denervated and radiated, the reconstructed breast displays shrinkage. A previous study reported that denervated flaps atrophy, but shrinkage has also been observed in innervated flaps without a statistically significant difference in the conventional latissimus dorsi myocutaneous flap [18]. As endoscopic partial reconstruction uses the latissimus dorsi muscle only, we can expect more shrinkage after denervation. Postoperative radiation therapy would result in further volume reduction. The reconstructed breast should be larger than the desired size to overcome postoperative asymmetry due to shrinkage of the radiated side. Further study is needed to determine the appropriate volume of overcorrection.

According to our experience, replacement of $20 \%$ to $40 \%$ of breast volume in the upper and lower outer quadrant with a latissimus dorsi muscle flap using endoscopic harvesting is a good alternative reconstruction technique after partial mastectomy. As this technique significantly reduces the incision size, less of the subcutaneous lymphatics and subdermal vascular plexus is injured, which theoretically leads to less edema, ecchymosis, seroma formation, and infection [19]. Guven et al. [20] reported a total postoperative drainage volume averaging $950 \mathrm{~mL}$, which is markedly less than those reported in previous standard latissimus dorsi muscle harvesting operations. Patients experience less pain and may experience easier movement of the ipsilateral upper extremity of the donor site [21]. A shorter incision requires less nursing care to the wound site and less dressing material. Therefore, the medical cost for postoperative wound care should be reduced. Although Lin et al. [21] reported no statistically significant difference between the classic harvesting technique and the endoscopic harvesting technique in the amount of intraoperative bleeding, postoperative hematoma, incidence of seroma, or infection in the donor site as assessed by the surgeon. Patients' attitudes and feelings about the scar and overall satisfaction were statistically significantly better in the endoscopic group.

On the other hand, this technique requires an intraoperative change in the patient's position, which elongates the operation time and increases the chances of infection [19]. A greater drawback is the limited visual field in the endoscope-assisted harvest of latissimus dorsi muscle flaps. To achieve an optimal optical cavity, several methods have been attempted, such as a designed tripod retraction device [16], retraction suture [17], manual retractor, balloon dissector [22], and gas insufflation [16,17], but these methods require operative dexterity and experienced skill. Development of customized, sophisticated instrumentation would be helpful, but the additional cost for such devices and increased complexity of the operating room set-up could be problematic. Furthermore, if mastectomy defects are large, the volume of transferred tissue for reconstruction may not be sufficient.

The options for treatment of breast cancer are numerous, and this approach-immediate reconstruction with endoscopic latissimus dorsi flap harvest-continues to gain popularity. We have performed 10 cases so far, so further studies should be performed with more patients and a longer period to produce more generalizable results evaluating the functional, oncological, and aesthetic outcomes of the operation.

\section{REFERENCES}

1. Fine NA, Schierle CF. Endoscopic approaches to the breast. In: Grotting JC, Neligan PC, editors. Plastic surgery. 3rd ed. New York: Elsevier Saunders; 2013. p.81-96.

2. Losken A, Hamdi M. Partial breast reconstruction: current perspectives. Plast Reconstr Surg 2009;124:722-36.

3. Pillarisetti RR, Querci Della Rovere G. Oncoplastic breast surgery. Indian J Surg 2012;74:255-63.

4. Park HS, Lee JS, Lee JS, et al. The feasibility of endoscopyassisted breast conservation surgery for patients with early breast cancer.J Breast Cancer 2011;14:52-7.

5. Missana MC, Pomel C. Endoscopic latissimus dorsi flap harvesting. Am J Surg 2007;194:164-9.

6. Adams WP Jr, Lipschitz AH, Ansari M, et al. Functional donor site morbidity following latissimus dorsi muscle flap transfer. Ann Plast Surg 2004;53:6-11.

7. Cho BC, Lee JH, Ramasastry SS, et al. Free latissimus dorsi muscle transfer using an endoscopic technique. Ann Plast Surg 1997;38:586-93.

8. Tan O, Aydin OE, Cinal H, et al. Latissimus dorsi flap harvest with a short incision. Microsurgery 2013;33:203-6.

9. Selber JC, Baumann DP, Holsinger FC. Robotic latissimus dorsi muscle harvest: a case series. Plast Reconstr Surg 2012; 129:1305-12.

10. Rainsbury RM, Paramanathan N. UK survey of partial mastectomy and reconstruction. Breast 2007;16:637-45.

11. Fisher B, Redmond C, Poisson R, et al. Eight-year results of a randomized clinical trial comparing total mastectomy and lumpectomy with or without irradiation in the treatment of breast cancer. N Engl J Med 1989;320:822-8.

12. Losken A. The oncoplastic approach to partial breast reconstruction. In: Grotting JC, Neligan PC, editors. Plastic surgery. 3rd ed. New York: Elsevier Saunders; 2013. p.296-313.

13. Boetes C, Mus RD, Holland R, et al. Breast tumors: comparative accuracy of MR imaging relative to mammography and US for demonstrating extent. Radiology 1995;197:743-7.

14. Brierley JD, Paterson IC, Lallemand RC, et al. The influence of breast size on late radiation reaction following excision 
and radiotherapy for early breast cancer. Clin Oncol (R Coll Radiol) 1991;3:6-9.

15. Cross MA, Elson HR, Aron BS. Breast conservation radiation therapy technique for women with large breasts. Int J Radiat Oncol Biol Phys 1989; 17:199-203.

16. Friedlander L, Sundin J. Minimally invasive harvesting of the latissimus dorsi. Plast Reconstr Surg 1994;94:881-4.

17. Fine NA, Orgill DP, Pribaz JJ. Early clinical experience in endoscopic-assisted muscle flap harvest. Ann Plast Surg 1994;33:465-9.

18. Szychta P, Butterworth M, Dixon M, et al. Breast reconstruction with the denervated latissimus dorsi musculocutaneous flap. Breast 2013;22:667-72.
19. Cha W, Jeong WJ, Ahn SH. Latissimus dorsi muscle free flap revisited: a novel endoscope-assisted approach. Laryngoscope 2013;123:613-7.

20. Guven E, Basaran K, Yazar M, et al. Electrothermal bipolar vessel sealer in endoscope-assisted latissumus dorsi flap harvesting. J Laparoendosc Adv Surg Tech A 2010;20:735-42.

21. Lin CH, Wei FC, Levin LS, et al. Donor-site morbidity comparison between endoscopically assisted and traditional harvest of free latissimus dorsi muscle flap. Plast Reconstr Surg 1999; 104:1070-7.

22. Karp NS, Bass LS, Kasabian AK, et al. Balloon assisted endoscopic harvest of the latissimus dorsi muscle. Plast Reconstr Surg 1997; 100:1161-7. 